\section{Bacterial gut shot}

\section{By Lev Osherovich, Senior Writer}

Enteric bacteria have long been suspected of helping maintain health, but the specific roles of these microbes have been hard to elucidate. Now, two independent American teams have linked the absence of normal gut bacteria with immune system abnormalities that lead to inflammation and allergy.

The findings are in line with the theory behind two new companiesEnterome Bioscience and Vedanta Biosciences Inc.- that aim to diagnose and treat inflammation arising from gut microbe deficiencies.

Both research teams looked at the effects of eliminating gut bacteria on immune system functioning in mice. A Boston researcher-led group found that mice lacking enteric bacteria early in life developed hyperactive invariant NK T (iNKT) cells that triggered inflammation in the colon and lungs and led to increased susceptibility to colitis and asthma. ${ }^{1}$

That group was co-led by Dennis Kasper, senior physician and professor of microbiology and immunology at Brigham and Women's Hospital and Harvard Medical School, and Richard Blumberg, professor of medicine and chief of the division of gastroenterology, hepatology and endoscopy at HMS and Brigham and Women's Hospital.

Separately, researchers led by David Artis, associate professor of microbiology and pathobiology at the Perelman School of Medicine at the University of Pennsylvania, found that killing off gut bacteria late in the life of adult mice led to a spike in allergy-associated basophils and IgE antibodies that caused allergy-like lung inflammation. ${ }^{2}$

In both cases, the findings connected for the first time allergic inflammation to the absence of normal microbes rather than to the presence of pathogenic gut bacteria.

Indeed, the discoveries provide a set of potential molecular and cellular mechanisms to explain the 'hygiene hypothesis', which states that the absence of early life exposure to normal microbes leads to a hyperactive immune system prone to allergy, inflammation and autoimmune disease.

"There's been a ton of intriguing observations and correlations between certain microbes and disease, but these papers really pin down the specific mechanisms and set the path for future work to identify therapeutic candidates," said Vedanta COO and PureTech Ventures senior associate Bernat Olle. These studies validate the hygiene hypothesis, which is now embraced by most allergists."
Vedanta is developing an oral formulation of enteric bacteria to correct the deficiency of normal bacteria in inflammatory bowel disease (IBD).

\section{Bugs out, cells in}

Blumberg and Kasper's team examined how the absence of bacteria in the environment affects the immune system of young mice.

The intestines of these germ-free mice had greater numbers of active iNKT cells in the colonic lamina propria, an epithelial layer near the gut surface, and in the lung than controls with normal gut bacteria.

iNKT cells are early immune sentinels that normally react to bacterial lipids, and inappropriate activation of the cells is thought to contribute to inflammatory indications and asthma. ${ }^{3}$ Indeed, the iNKT cells made the mice susceptible to experimentally induced intestinal inflammation and asthma.

In a chemically induced model of ulcerative colitis, germ-free mice had higher proinflammatory cytokine levels, gut inflammation and mortality than similarly treated mice with normal gut bacteria. The team saw similar results in an ovalbumin (OVA)-induced model of asthma.

"The paper shows that germ-free mice, which are born in germ-free environments with germ-free mothers, were very susceptible to two experimentally induced diseases mediated by iNKT cells," said Kasper. "Normal mice born to colonized mothers are resistant to these diseases. Thus, the bacteria make the mice resistant to these diseases."

Results were published in Science and were not patented.

The UPenn team performed a study in mice reared in a germ-free environment and in adult mice that received multiple antibiotics to kill the majority of their enteric bacteria. Both sets of mice had greater circulating levels of proinflammatory basophils and IgE than untreated controls and showed histological evidence of airway inflammation.

Results were published in Nature Medicine. The patent and licensing status of the work was not disclosed.

\section{More mechanism needed}

Although the two studies point to specific immune response mechanisms that go awry in the absence of bacteria, further work is needed to connect the findings to human disease and identify therapeutic intervention points.

Enterome CEO Pierre Belichard said the findings are evidence that early exposure to gut microbes helps establish normal immunity.

"When you are born, you have absolutely no bacteria in the gut and are invaded by the bacteria within the first few hours of life, and the immune system adapts to these organisms," said Belichard.

Enterome is developing diagnostics for microbial imbalances in IBD and nonalcoholic fatty liver disease (NAFLD). In March, the company raised $€ 5$ million ( $\$ 6.6$ million) in a series A financing to develop 


\section{ANALYSIS}

its discovery-stage IBD diagnostic and conduct a clinical trial of its NAFLD test.

Although local inflammatory effects due to gut microbial abnormalities have been seen in multiple prior studies by other researchers, Olle said it was "interesting that both of these papers also deal with allergy, which is at the periphery. This helps to support the idea that microbes in our gut can affect distal organ systems" including the lungs.

Kasper and Blumberg's team is operating under the hypothesis that the absence of inhibitory signals from gut microbes causes iNKT cells, basophils and other immune system components to become hyperactive. The group now is looking for the specific molecules produced by bacteria that tame iNKT cells and for the cellular receptors for these molecules.

The team has found a candidate-chemokine CXC motif ligand 16 (CXCL16), a proinflammatory cytokine involved in iNKT cell activation. In the Science paper, the team reported that immunodepletion of CXCL16 decreases iNKT levels in the lung and colon in germ-free mice compared with control antibody treatment.

Once the specific molecules and pathways that trigger the proinflammatory iNKT cells, basophils and IgE antibodies are found, it should be possible to figure out the order of events that lead to inflammation and test whether these pathways play a role in human disease.
Kaspar said he is open to collaborating with industry to further characterize the role of CXCL16 and other factors in gut inflammation and allergy.

Osherovich, L. SciBX 5(14); doi:10.1038/scibx.2012.353

Published online April 5, 2012

\section{REFERENCES}

1. Olszak, T. et al. Science; published online March 22, 2012; doi:10.1126/science.1219328

Contact: Richard S. Blumberg, Brigham and Women's Hospital and Harvard Medical School, Boston, Mass.

e-mail: rblumberg@partners.org

Contact: Dennis L. Kasper, same affiliation as above e-mail: dennis_kasper@hms.harvard.edu

2. Hill, D.A. et al. Nat. Med.; published online March 25, 2012; doi:10.1038/nm.2657

Contact: David Artis, Perelman School of Medicine at the University of Pennsylvania, Philadelphia, Pa. e-mail: dartis@mail.med.upenn.edu

3. Iwamura, C. \& Nakayama, T. Curr. Opin. Immunol. 22, 807-813 (2010)

COMPANIES AND INSTITUTIONS MENTIONED

Brigham and Women's Hospital, Boston, Mass.

Enterome Bioscience, Paris, France

Harvard Medical School, Boston, Mass.

Perelman School of Medicine at the University of Pennsylvania, Philadelphia, Pa.

PureTech Ventures, Boston, Mass.

Vedanta Biosciences Inc., Boston, Mass. 